\title{
One year changes in disability in multiple sclerosis: neurological examination compared with patient self report
}

\author{
E L J Hoogervorst, M J Eikelenboom, B M J Uitdehaag, C H Polman
}

J Neurol Neurosurg Psychiatry 2003;74:439-442

$\mathrm{T}$ he most common neurological outcome measure for multiple sclerosis (MS) patients is the Expanded Disability Status Scale (EDSS). Originally, Kurtzke developed this disability status scale, ${ }^{1}$ later revised to a more refined classification system, known as the EDSS. ${ }^{2}$ The EDSS is based on a neurological examination of eight functional systems usually performed by a medical doctor. While problems of standardisation, resulting in suboptimal interrater reliability, marginal sensitivity to change and bias to locomotor function have been described $^{3-6}$ the EDSS remains a useful tool for classifying disability in MS patients.

In a longstanding invalidating neurological disease like MS where cure is not yet possible, disability as perceived by the patient is an important measure and for this purpose the Guy's Neurological Disability Scale (GNDS) ${ }^{7}$ was recently developed. The GNDS measures disability based on patient self report, embraces the whole range of disabilities that can be encountered in the course of MS, is patient orientated, user friendly, and not biased to any particular disability. ${ }^{78}$ The GNDS is a questionnaire, divided in 12 subcategories, directed to assess a patient's disability in the previous month by patient interview and can be applied by any healthcare worker. It is therefore extremely practical and capable of incorporating patients' views of their disability in a structured manner. ${ }^{89}$

In a previous study we showed good cross sectional correlations between EDSS and GNDS: these correlations were mainly attributable to the importance of spinal cord related neurological functions for both scales.' That study also confirmed that the GNDS measures other dimensions of disability than the EDSS and incorporates patients' perspectives, both of which can cause marked discrepancies between scores on the two scales. Such discrepancies between objective measurements and subjective complaints have been demonstrated in many studies. To give just one example, Sharrack and Hughes found that the assessment of a patient's walking ability is very different between doctors and patients, and also very different from measured distances. ${ }^{10}$

The aim of this study was to prospectively characterise the relation between one year changes in neurologist rating of neurological examination of abnormalities as measured by the EDSS and changes in patient self report as measured by the GNDS. More specifically, we studied whether discrepancies between changes in EDSS and GNDS were attributable to differences in the change perceived by the patient and that measured by the physician or the result of symptoms covered by the GNDS but not included in the EDSS. In addition, we studied how significant changes in the EDSS were associated with changes in patient perceived disability.

\section{METHODS}

\section{Patients}

Two hundred and fifty patients with clinically definite $\mathrm{MS}^{11}$ underwent longitudinal examinations of EDSS and GNDS at our outpatient clinic. Patients were diagnosed as having relapsing-remitting MS $(n=126)$, secondary progressive MS $(n=60)$, or primary progressive MS $(n=64) .{ }^{12}$

\section{Test procedures}

Disability was assessed at baseline and after one year using EDSS and GNDS. Data from EDSS and GNDS were collected in the same visit, under standardised conditions by well trained physicians. The two measurements were performed in the same order, first the GNDS followed by the EDSS.

The EDSS is divided in 20 half steps ranging from 0 (normal) to 10 (death due to MS). ${ }^{2}$ Each subcategory of the GNDS was scored separately ranging from 0 (normal) to 5 (maximum help required). The GNDS score is the sum score of the 12 subcategories (range 0 to 60 ). ${ }^{7}$

\section{Analysis}

Results were analysed in several ways. We studied cross sectional correlations between EDSS and GNDS sum score at baseline and follow up. Longitudinal correlations were studied between $\Delta$ EDSS and $\Delta$ GNDS sum score, $\Delta$ functional systems, and $\Delta$ GNDS subcategories, as well as between $\Delta$ functional systems and the corresponding $\Delta$ GNDS subcategories ( $\Delta$ sphincter compared with $\Delta$ bladder and bowel function; $\Delta$ brain stem compared with $\Delta$ speech and swallowing; $\Delta$ pyramidal, sensory and cerebellar compared with $\Delta$ upper and lower limb function; $\Delta$ cerebral compared with $\Delta$ cognition and mood and $\Delta$ vision compared with $\Delta$ visual). ${ }^{9}$

As this analysis may be confounded by the fact that the EDSS is an ordinal scale, we also studied the total number of patients showing a clinically significant change on the EDSS and GNDS, as well as the mean change in GNDS for patients with a change on the EDSS. A clinically significant change of the EDSS was defined as a change of 1.0 point or more at EDSS levels $<5.5$ or 0.5 point or more at EDSS levels $\geqslant 5.5 .^{13}$ For the GNDS a change of three or more points in the sum score was considered as clinically significant. ${ }^{74}$ As the threshold of significance is not well defined we performed a sensitivity analysis in which we defined a significant change on the GNDS as a change of at least 2, 4, and 5 points, as well.

\section{Statistics}

Correlations were calculated using the Spearman rank correlation coefficient $(r)$. In this study we considered $\mathrm{p}$ values of less than 0.01 as significant and $p$ values of less than 0.05 as a trend only. The strength of correlation was labelled as follows: correlations $<0.40$ as weak to marginal; 0.40 to 0.60 as moderate; 0.60 to 0.80 as good, and $>0.80$ as excellent. Agreement between clinically significant EDSS and GNDS changes was calculated using linear weighted Cohen's $\kappa$.

Abbreviations: MS, multiple sclerosis; EDSS, Expanded Disability Status Scale; GNDS, Guy's Neurological Disability Scale 
Table 1 GNDS sum score expressed as median (interquartile range)

\begin{tabular}{ll}
\hline & Total \\
\hline Total & 250 \\
$M$ & 95 \\
$F$ & 155 \\
Age (y) & $44.3(11.0)$ \\
Baseline EDSS & $4.0(2.5-6.0)$ \\
Follow up EDSS & $4.0(2.9-6.0)$ \\
$\Delta$ EDSS & $0.0(0.0-0.5)$ \\
Baseline GNDS ${ }^{2}$ & $13.0(8.0-19.0)$ \\
Follow up GNDS & $14.0(9.0-20.0)$ \\
$\Delta$ GNDS & $1.0(-2.0-3.0)$ \\
\hline
\end{tabular}

EDSS, Expanded Disability Status Scale; GNDS, Guy's Neurological Disability Status Scale.

Table 2 Correlations between $\Delta$ GNDS subcategories and $\Delta$ EDSS and $\triangle$ GNDS for the total population

\begin{tabular}{lll}
\hline$\Delta$ GNDS subcategories & $\Delta$ EDSS & $\Delta$ GNDS \\
\hline Cognition & $0.06^{\text {ns }}$ & $0.29^{* *}$ \\
Mood & $0.08^{\text {ns }}$ & $0.48^{* *}$ \\
Vision & $0.03^{\text {ns }}$ & $0.11^{\text {ns }}$ \\
Speech & $0.15^{*}$ & $0.20^{* *}$ \\
Swallowing & $-0.06^{\text {ns }}$ & $0.09^{\text {ns }}$ \\
Upper limb function & $0.11^{\text {ns }}$ & $0.33^{* *}$ \\
Lower limb function & $0.28^{* *}$ & $0.30^{* *}$ \\
Bladder function & $0.08^{\text {ns }}$ & $0.43^{* *}$ \\
Bowel function & $-0.02^{\text {ns }}$ & $0.23^{* *}$ \\
Sexual function & $-0.01^{\text {ns }}$ & $0.41^{* *}$ \\
Fatigue & $0.16^{\text {ns }}$ & $0.34^{* *}$ \\
Others & $-0.07^{\text {ns }}$ & $0.40^{* *}$ \\
\hline
\end{tabular}

** Correlation is significant $(p<0.01)$; "correlation shows a trend $(p<0.05) ;{ }^{\text {ns }}$ not significant. Abbreviations as for table 1 .

\section{RESULTS}

Table 1 summarises patient characteristics and scores on EDSS and GNDS at baseline and follow up. Mean age at baseline was 44.3 years (SD 11.0); $38 \%$ of the patients were male and $62 \%$ were female. Average time interval from baseline to follow up measurement was 13.6 months (SD 1.9). Baseline EDSS scores ranged from 0 to 9.0 and median EDSS scores at baseline and follow up were the same (4.0). The median GNDS sum score at follow up (14.0) showed a deterioration of 1.0 point compared with baseline (13.0).

Changes in GNDS occurred independent from baseline GNDS sum scores; both frequency and magnitude of the $\Delta$ GNDS were equally distributed along the full range of baseline GNDS sum scores, whereas changes in EDSS were more frequent and larger in the lower part of the scale (data not shown). This emphasises an important measurement scale characteristic of the GNDS.

Cross sectional correlations between EDSS and GNDS sum score were $0.69(\mathrm{p}<0.01)$ at baseline and $0.77(\mathrm{p}<0.01)$ at follow up. With respect to changes over time, only a weak to marginal correlation was found between $\Delta$ EDSS and $\Delta$ GNDS sum score $(r=0.19 ; \mathrm{p}<0.01)$.

Correlations between change in the EDSS functional systems and change in the corresponding GNDS subcategories were marginal to weak ( $\Delta$ brain stem compared with $\Delta$ speech and swallowing, $r=0.12$, not significant; $\Delta$ pyramidal, sensory and cerebellar compared with $\Delta$ upper and lower limb function, $r=0.16, \mathrm{p}<0.05 ; \Delta$ cerebral compared with cognition and mood, $r=0.25, \mathrm{p}<0.01$ and $\Delta$ vision compared with visual $r=0.23, \mathrm{p}<0.01)$. A moderate correlation was found between $\Delta$ sphincter of the EDSS and $\Delta$ bladder and bowel function of the GNDS $(r=0.58, \mathrm{p}<0.01)$.
Table 3 Correlations between $\Delta$ functional systems and $\Delta$ EDSS and $\Delta$ GNDS for the total population

\begin{tabular}{lll}
\hline$\Delta$ Functional systems & $\Delta$ EDSS & $\Delta$ GNDS \\
\hline Visual & $0.13^{\text {ns }}$ & $0.08^{\text {ns }}$ \\
Brain stem & $0.12^{\text {ns }}$ & $0.18^{* *}$ \\
Pyramidal & $0.22^{* *}$ & $0.06^{\text {ns }}$ \\
Sensory & $0.30^{* *}$ & $0.01^{\text {ns }}$ \\
Cerebellar & $0.21^{* *}$ & $-0.00^{\text {ns }}$ \\
Sphincter & $0.12^{\text {ns }}$ & $0.34^{* *}$ \\
Cerebral & $0.13^{*}$ & $0.26^{* *}$ \\
\hline
\end{tabular}

** Correlation is significant $(p<0.01)$; "correlation shows a trend $(p<$ $0.05) ;{ }^{\text {ns }}$ not significant. Abbreviations as for table 1.

Table 4 Number of patients showing clinically significant changes in EDSS and GNDS sum score

\begin{tabular}{llll}
\hline & \multicolumn{3}{l}{ Significant $\Delta$ EDSS } \\
\cline { 2 - 4 } $\begin{array}{l}\Delta \text { GNDS of } 3 \\
\text { points or more }\end{array}$ & $\begin{array}{l}\text { Improvement } \\
n=37\end{array}$ & $\begin{array}{l}\text { No change } \\
n=154\end{array}$ & $\begin{array}{l}\text { Worsening } \\
n=59\end{array}$ \\
\hline $\begin{array}{l}\text { Improvement } n=52 \\
\text { No change } n=112\end{array}$ & 12 & 31 & 9 \\
Worsening $n=86$ & 11 & 75 & 23 \\
\hline Abbreviations as for table 1. & 48 & 27 \\
\hline
\end{tabular}

Table 2 shows that the only statistically significant correlation between change in the different GNDS subcategories and $\Delta$ EDSS was found for lower limb function, whereas (as expected) many more GNDS subcategories were correlated with $\Delta$ GNDS. Table 3 shows that of all functional systems only changes in brain stem, sphincter, and cerebral functional systems correlated significantly with changes in GNDS sum score, while with respect to the EDSS the situation is opposite, with the exception of the visual system, which is not correlated to either overall score.

Table 4 shows that a total of 59 patients showed a clinically significant worsening on the EDSS, whereas 37 patients showed a clinically significant improvement. A clinically significant worsening or improvement of the GNDS sum score (based on the definition of a change of at least three points being significant) was observed in 86 and 52 patients, respectively. Strikingly, opposite changes in EDSS compared with GNDS sum score were observed in 20 patients $(8 \%)$ : in 11 patients of $37(29.7 \%)$ in whom there was a clinically significant improvement on the EDSS there was a 3 point or more worsening in the GNDS, whereas, vice versa, in 9 patients of 59 $(15.2 \%)$ in whom there was a clinically significant worsening on the EDSS there was a 3 point or more improvement on the GNDS sum score. Poor agreement (linear weighted Cohen's $\kappa=0.12$ ) was found between change in disability as measured by neurologist rating of neurological examination abnormalities and patient self report.

Varying the definition of significant change in GNDS to at least 2,4 , or 5 points resulted in corresponding percentages of patients with a significant worsening on the EDSS accompanied by a significant improvement on the GNDS of $22.0 \%$ ( 13 of 59 ), $10.2 \%$ (6 of 59), and $10.2 \%$ (6 of 59) respectively. Agreement between the two disability measures remained poor with respective linear weighted Cohen's $\kappa$ values of 0.11 , 0.13 , and 0.16 .

Figure 1 shows the mean GNDS change per category of EDSS change: the profile of changes clearly indicates that for patients with a significant improvement on the EDSS there is the largest improvement on the GNDS and vice versa that for patients with a significant worsening on the EDSS there is the largest worsening on the GNDS. 


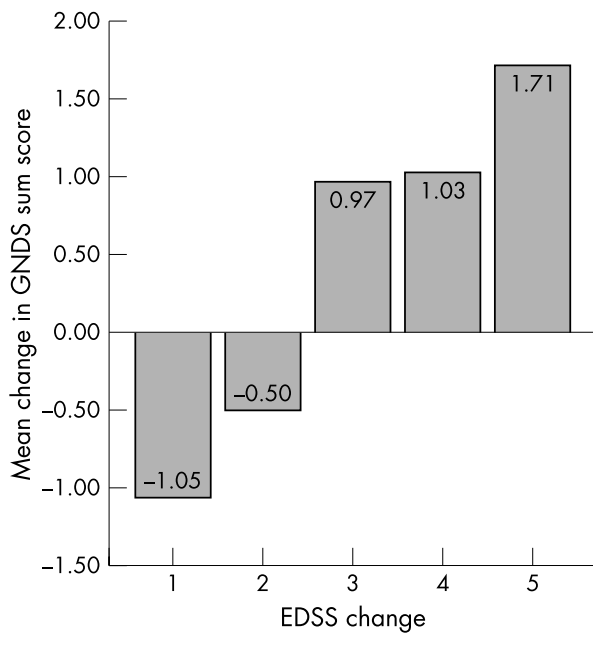

Figure 1 Mean change in GNDS sum score per (significant) EDSS change. 1, Significant EDSS improvement; 2, not significant EDSS improvement; 3, no EDSS change; 4, not significant EDSS worsening; 5, significant EDSS worsening.

\section{DISCUSSION}

Assessment of the patient's perspective of disability is important, as reflected by the increasing number of studies incorporating such measures. ${ }^{15}$ To our knowledge, until now, no other large study has reported on the relation between change in neurologist rating of neurological examination abnormalities as measured by the EDSS and change in patient perceived disability.

Two important pieces of information can be obtained from the data collected in this study. In the first place we show that the correlation between one year longitudinal changes in the EDSS compared with GNDS is substantially lower than the cross sectional correlation between these measures; in the second place we show that, dependent on the criterion applied for significant change in the GNDS, $10 \%$ to $22 \%$ of the patients who have a significant worsening on the EDSS show a significant improvement in perceived disability.

It is obvious from our data that despite the profile of mean GNDS sum score changes being nicely rank ordered in relation to EDSS changes (significant or not according to commonly applied criteria, fig 1), the longitudinal correlation between EDSS and GNDS is much lower than the cross sectional correlations. Although this is partially attributable to the magnitude of one year changes being considerably smaller than the range of scores at a given point in time, detailed analyses of our data also gives rise to some other explanations. Correlations between changes in functional systems and changes in GNDS subcategories are only moderate to good for those functional systems that are (mainly) based on patient self report; those functional systems that are based on neurological examination are poorly or not at all correlated with the subcategory scores based on the patient's perception. Table 3 shows that whereas changes in the EDSS are only correlated to changes in lower limb function, changes in the GNDS are significantly correlated to a wide range of disability subcategories, including categories that theoretically could have lead to EDSS changes (bladder function and bowel function) and categories that are not at all incorporated in the EDSS (that is, fatigue).

In conclusion there seem to be three reasons for the discrepancies between changes in EDSS and GNDS: (1) differences in the change perceived by the patient and that measured by the physician, (2) changes in many disability subcategories not leading to changes in EDSS, and (3) the GNDS incorporating aspects of disability (for example, fatigue) that are not at all considered by the EDSS. Of course it is well known from other studies that the EDSS is heavily biased to locomotor function, ${ }^{3}{ }^{4}$ but this study adds a unique dimension based on how disability is perceived by MS patients.

Next, to compensate for the fact that our results might have been influenced by the changes in disability that occurred over a follow up period of about one year being comparatively small, we performed additional analyses on those patients who showed significant EDSS changes. For these analyses we used widely applied definitions for significant EDSS change. ${ }^{13}$ In many recent clinical trials in MS this definition of a significant change has been used to define significant worsening that served as primary outcome measure to determine whether disease modifying interventions were effective or not. ${ }^{16-18}$ In an attempt to obtain information on individual patients we also analysed how many patients with significant EDSS changes have corresponding or opposite changes in the GNDS of at least 2, 3, 4, or 5 points. Based on the data by Sharrack and Hughes ${ }^{79}$ we defined a change of at least three points to be primary for this purpose; clinically significant worsening in the physician derived EDSS was accompanied by a clinically significant increase in disability as perceived by the patient in only less than $50 \%$ of patients. Remarkably, $15 \%$ of these patients in whom a clinically significant worsening of EDSS was documented reported a significant improvement in their own subjective perception of disability compared with $30 \%$ who reported a significant worsening whereas a significant improvement in EDSS was found.

Performing these analyses for GNDS changes of at least 2, 4, and 5 points the percentages of patients showing a significant worsening on their EDSS with an associated improvement on the GNDS would have been $22 \%, 10 \%$, and $10 \%$ respectively, indicating that this phenomenon is not dependent on the specific cut off of GNDS change.

In our opinion it is highly unlikely that our observations are attributable to measurement errors with respect to the EDSS: EDSS scores were obtained by a restricted number of physicians who had undergone multiple training sessions. In addition, the percentage of patients in this study showing significant worsening in EDSS score over one year $(24 \%)$ is very similar to that in many other studies..$^{79-21}$ Of course the patient's perspective is subjective by nature and may change over time without corresponding objective changes. This may lead to response shift and thus complicate the comparison between changes in GNDS and EDSS.

In conclusion, we present longitudinal data supporting the idea that patient's impression of change in disability may differ not just quantitatively but also qualitatively from that of an examining physician. This seems to be because there are true differences in the change perceived by the patient and that measured by the physician and to the fact that many more dimensions of disability have an impact on the GNDS than on the EDSS. Longer follow up of our cohort and additional studies in other patient groups are required to better understand this phenomenon and to determine what consequences it can have for future clinical trial design.

\section{Authors' affiliations}

E L J Hoogervorst, M J Eikelenboom, B M J Uitdehaag, C H Polman, Department of Neurology, VU Medical Centre,

Amsterdam, Netherlands

B M J Uitdehaag, Department of Clinical Epidemiology and Biostatistics, VU Medical Centre

Competing interests: none declared.

Correspondence to: Dr E L J Hoogervorst, Department of Neurology, VU Medical centre, Academisch Ziekenhuis Vrije Universiteit, Postbox 7057, Amsterdam 1007 MB, Netherlands; e.hoogervorst@vumc.nl

Received 13 August 2002

Accepted in revised form 21 December 2002 


\section{REFERENCES}

1 Kurtzke JF. A new scale for evaluating disability in multiple sclerosis. Neurology 1955:5:580-3.

2 Kurtzke JF. Rating neurologic impairment in multiple sclerosis: an expanded disability status scale (EDSS). Neurology 1983;33:1444-52.

3 Whitaker JN, McFarland HF, Rudge P, et al. Outcomes assessment in multiple sclerosis clinical trials: a critical analysis. Multiple Sclerosis 1995; 1:37-47.

4 Noseworthy JH. Clinical scoring methods for multiple sclerosis. Ann Neurol 1994;36 (suppl):S80-5.

5 Hobart J, Freeman J, Thompson A. Kurtzke scales revisited: the application of psychometric methods to clinical intuition. Brain 2000:123: 1027-40.

6 Sharrack B, Hughes RA, Soudain S, et al. The psychometric properties of clinical rating scales used in multiple sclerosis. Brain 1999;122:141-59.

7 Sharrack B, Hughes RA. The Guy's Neurological Disability Scale (GNDS): a new disability measure for multiple sclerosis. Mult iple Sclerosis 1999;5:223-33.

8 Sharrack B, Hughes RA. Scale development and Guy's Neurological Disability Scale. J Neurol 1999; 246:226.

9 Hoogervorst ELJ, van Winsen LM, Eikelenboom M, et al. Comparisons of patient self-report, neurologic examination, and functional impairment in MS. Neurology 2001;56:934-7.

10 Sharrack B, Hughes RA. Reliability of distance estimation by doctors and patients: cross sectional study. BMU 1997;315:1652-4.

11 Poser CM, Paty DW, Scheinberg L, et al. New diagnostic criteria for multiple sclerosis: guidelines for research protocols. Ann Neurol 1983:13:227-31.

12 Lublin FD, Reingold SC. Defining the clinical course of multiple sclerosis: results of an international survey. National Multiple Sclerosis Society (USA) Advisory Committee on Clinical Trials of New Agents in Multiple Sclerosis. Neurology 1996;46:907-11.
13 Goodkin DE. EDSS reliability. Neurology 1991;41:332.

14 Hoogervorst EL, Kalkers NF, van Winsen LML, et al. Differential treatment effect on measures of neurologic exam, functional impairment and patient self-report in multiple sclerosis. Multiple Sclerosis $2001 ; 7: 335-9$.

15 Freeman JA, Thompson AJ, Fitzpatrick R, et al. Interferon-beta lb in the treatment of secondary progressive MS: impact on quality of life. Neurology 2001;57:1870-5.

16 Secondary Progressive Efficacy Clinical Trial of Recombinant Interferon-beta-la in MS (SPECTRIMS) Study Group. Randomized controlled trial of interferon-beta-la in secondary progressive MS Clinical results. Neurology 2001;56:1496-504.

17 Jacobs LD, Cookfair DL, Rudick RA, et al. Intramuscular interferon beta-l a for disease progression in relapsing multiple sclerosis. The Multiple Sclerosis Collaborative Research Group (MSCRG). Ann Neurol 1996;39:285-94.

18 European Study Group on interferon beta-1b in secondary progressive MS. Placebo-controlled multicentre randomised trial of interferon beta- $1 \mathrm{~b}$ in treatment of secondary progressive multiple sclerosis. Lancet 1998;352:1491-7.

19 Weinshenker BG, Rice GP, Noseworthy JH, et al. The natural history of multiple sclerosis: a geographically based study. 4. Applications to planning and interpretation of clinical therapeutic trials. Brain 1991;114:1057-67.

20 Hohol MJ, Orav EJ, Weiner HL. Disease steps in multiple sclerosis: a longitudinal study comparing disease steps and EDSS to evaluate disease progression. Multiple Sclerosis 1999;5:349-54.

21 Stevenson VL, Miller DH, Leary SM, et al. One year follow up study of primary and transitional progressive multiple sclerosis. J Neurol Neurosurg Psychiatry 2000;68:713-18.

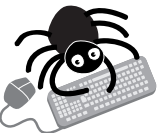

Please visit the Journal of Neurology, Neurosurgery, and Psychiatry website [www.jnnp.com/ supplemental] for link to this full article.

\section{Symptoms can predict the need for computed tomography in minor head} injury

A method that uses common odds ratios to predict risk of abnormality from symptoms could help doctors to provide more appropriate care for minor head injury.

Pooled odds ratios from published case-control studies of patients with minor head injury (Glasgow coma scale (GCS) 15) with loss of consciousness (LOC) or post traumatic amnesia (PTA) indicated that just three symptoms predicted abnormality on computed tomography. They were vomiting (odds ratio $4.398 ; 95 \%$ confidence interval 2.790 to 6.932 ), nausea (2.125; 1.467 to 3.057$)$, and severe headache $(3.211 ; 2.212$ to 4.584$)$; blurred vision and dizziness were not significant. This finding enabled patients to be subgrouped as: GCS 15a very small risk (no symptoms); GCS 15b low risk (mild headache/blurred vision/dizziness); GCS 15c intermediate risk (LOC/PTA); GCS 15d high risk (severe headache/nausea/vomiting).

The authors carried out a meta-analysis of case-control or nested case-control studies to calculate common odds ratios for five symptoms and abnormality on computed tomography. They searched Medline and PubMed between January 1990 and February 2001 and hand searched several journals. They included only full papers of studies of adults or adults and children with LOC/PTA, all of whom had been examined by computed tomography, whose data allowed common odds ratios to be calculated. Just three papers, covering 3375 patients, met the study criteria.

Minor head injury has most recently been classified as GCS 15, but it still needs to be separated from other injuries within this group. Individual studies that have tried to link symptoms to abnormality on computed tomography are nearly all flawed in some way.

^ Emergency Medicine Journal 2002;19:515-9. 


\section{CORRESPONDENCE}

\section{The naming of parts}

Many deplore the journalistic trend to label well recognised conditions by acronyms, or by recently invented names-commonly to no useful purpose. Thus neurologists may not welcome yet another two names, recorded in past literature but not in general currency.

Umapathi et al give a most valuable and timely review of the dropped head and bent spine syndromes. ${ }^{1}$ But why perpetuate the ungainly phrase head ptosis? Ptosis, Greek $\pi \tau \omega \sigma \mathrm{s} \varsigma=$ falling, has traditionally been applied only to the upper eyelid and to prolapse of any of the viscera or of the breasts. Head drop is short and its meaning is unequivocal.

Camptocormia, Greek $\kappa \alpha \mu \pi v \lambda=$ bent $\kappa о \rho \mu о \varsigma=$ trunk of a tree: but, like a few others, ${ }^{2}$ Umapathi et al apply it to signify a bent spine. Camptocormia was first an illnes occurring among soldiers in World Wars I and II, and was regarded as a sign of hysteria. Ankylosing spondylitis is a more frequen cause. It is an ostentatious word that portends something more mysterious than a bent back or neck. To be similarly pretentious, can we not survive without such euphuisms?

J M S Pearce Anlaby, East Yorks, UK; imsp@freenet.co.uk

\section{References}

1 Umapathi T, Choudhry V, Cornblath D, et al. Head drop and camptocormia. I Neuro Neurosurg Psychiatry 2002;73:1-8.

2 Sinel M, Eisenberg MS. Two unusual gait disturbances: astasia, abasia and camptocormia. Arch Phys Med Rehabil 1990;71:1078-80.

\section{Head drop and camptocormio}

The article by Umapathi $e t$ al ${ }^{1}$ in this journal referred to the original use of the term camptocormia by Souques in 1915, though functional bent back was first described by Brodie in 1837. Mlle Rosanoff-Saloff supported Souques' case study with a photographic record of this soldier's bent back and his recovery. According to the English translation abstract in Southard's fine collection of shell shock cases $^{3}$ this soldier was wounded five month previously by a bullet that entered along the auxiliary border of the scapula and emerged near the spine. "He spat blood for several days ... and when he got up his trunk and thighs were found to be in a state of moderate flexion upon the pelvis, the trunk being bent almost at a right angle." He was able to bend his trunk still further forward than its habitual contractured position' and it was evident that there was contraction of the muscles of the abdominal wall and of the iliopsoas. "No motor, sensory, reflex, trophic, vasomotor, electrical, visceral or X-ray disorders could be found." The application of plas ter corsets 'cured' this man's deformity within six weeks.

The poilus spoke of this condition as cintrage (arching), suggesting that it was not an uncommon affliction of the French soldier. Seemingly only recorded by French neurologists, Roussy and Lhermitte reported two subsequent cases. ${ }^{3}$ An infantryman was thrown into the air by the bursting of a shell, rendered unconscious and recovered experiencing violent pains in the back. He remained stooped to the right. His bent back was corrected by the application of plaster corsets. The other reported case was that of a chasseur who was buried in an explosion, knocked unconscious, and experienced acute respiratory distress, and subsequent mutism and camptocormia. One séance of electrical treatment corrected the improper attitude of the trunk, though he did continue to experience "a few persistent lumbar pains".

It would be difficult to doubt the probability that psychological factors influenced these men's recuperation. To describe these soldiers as hysterical, ${ }^{1}$ though this was the terminology used during this period, or indeed that they suffered functional bent back, is probably unfair. They may well have suffered acute traumatic spinal injury and reactive muscle spasm (and contractures). Persistent stooping in shallow trenches, in appalling conditions of deprivation and danger, may have been contributing factors weakening the tone of paraspinal muscles. However, these case reports suggest that the traumatic injury alone may be sufficient explanation for the bent spines. The management of camptocormia in the first world war was to provide biomechanical supports, such as corsets, apparently with good results. The psychological therapies of "persuasive reeducation" were additive rather than pivotal and faradisation (and other tortures) used only "if necessary". ${ }^{3}$

The Sandler triad of low self esteem with confusion of identity, sadomasochistic behaviour toward military authorities, and impotence ${ }^{4}$ were, in 1947, proposed as being an essential part of camptocormia. Umapathi's ${ }^{1}$ recognised causes of camptocormia and the contributing factors however implicate organicity, as indeed do the original case reports.

A D Macleod Psychiatric Consultation Service, Christchurch Hospital, Private Bag 4710, Christchurch, New Zealand; PCS@cdhb.govt.nz

\section{References}

1 Umapathi T, Chaudhry V, Cornblath D, et al. Head drop and camptocormia. J Neurol Neurosurg Psychiatry 2002;73: 1-7.

2 Souques A, Rosanoff-Saloff M. La camptocormie. Rev Neurol (Paris) 1914;22:937-9.

3 Southard EE. Shell-shock and other neuropsychiatric problems presented in 589 case histories from the war literature, 1914-1918. Boston: WM Leonard, 1919

4 Sandler SA. Camptocormia, or the functional bent back. Psychosom Med

1947;9: 197-204.

\section{Author's reply}

We would like to thank Dr J M S Pearce for his comments.

We agree with him on the proliferation of medical terms referring to similar if not identical conditions. One of the chief aims of writing this paper is to thread a line of commonality through the various names in literature, which in essence refer to an anterior curvature of the spine. Hence the title "Head drop and camptocormia, the spectrum of bentspine disorders".

However, we would like to disagree with Dr Pearce labelling the spinal deformity seen in ankylosing spondylitis as camptocormia. In arthritic conditions and diseases that affect bone, the spinal deformity is fixed. In the bent-spine disorders referred to in the paper, the deformity may reduce considerably or even disappear with change in position, for example when supine. We would therefore prefer to reserve the phrases head drop (used interchangeably with head ptosis) and camptocormia to neurological conditions that affect the strength or tone of the muscles controlling spinal posture.

As aficionados of medical history, we very much enjoy Dr A D Macleod's letter. We agree that organic factors might have contributed to the camptocormia in solders believed to have been suffering from hysteria. It would have not been unexpected for patients, like the man described by Southard with a bullet wound near the spine, ${ }^{1}$ to have developed spasm or even denervation of thoracic paraspinal muscles.

T Umapathi

Department of Neurology, National Neuroscience Institute, 11 Jalan Tan Tock Seng, 308433 Singapore; tumapathi@yahoo.com

\section{Reference}

1 Southard EE. Shell shock and other neuropsychiatric problems presented in 589 case histories from the war literature 1914-1918. Boston: WM Leonard, 1919.

\section{Infection and multiple sclerosis}

The article by Hawkes ${ }^{1}$ and the editorial commentary about the role of infectious agents in multiple sclerosis (MS) examined this question from a new viewpoint based on epidemiological observations. ${ }^{2}$ Several infectious agents, most not sexually transmitted, were reported to be associated with MS according to epidemiological data, serology in CSF and blood, or demonstration of pathogens in tissue. A relation with measles virus (MV) has been an early and most consistent finding. More recently, higher prevalence and higher titres of antibodies against human herpesvirus 6 (HHV6), but not other herpesviruses, were shown in MS patients compared to control groups, suggesting different exposure to HHV6 in MS. ${ }^{3}$ HHV6, like vaccine strain $M V$ and certain wild type $M V$, uses the membrane cofactor protein (MCP; CD46) as a receptor for entry into cells. This suggests a possible involvement of CD46 in MS.

The possibility of a particular isoform of CD46 predisposing MS patients to infection is unlikely because all isoforms have similar affinity to MV. Increased levels of soluble CD46 have been reported in the serum and cerebrospinal fluid of MS patients, more in those who have HHV6 DNA. ${ }^{4}$ One interpretation of these findings involved increased activity of the complement system in MS. However, experimental studies show no influence of inflammatory cytokines on CD46 expression and do not support inflammation 
as a cause of increased CD46. Incorporation of CD46 in the viral envelope, or a possible genetic propensity in MS patients, have also been considered as causes of increased CD46. ${ }^{4}$ While its origin in MS is unclear, soluble CD46 might be involved in viral pathogenesis by binding the virus in the viraemic phase and allowing another to attach to CD46 and spread from cell to cell. Both HHV6 and MV are infectious agents encountered in early childhood, and HHV6 can indeed become reactivated a few weeks after primary MV infection. On the other hand, because HHV6 and MV downregulate CD46 expression on the infected cell, they may diminish the entry of each other, delaying the time of infection. Therefore, they might produce increased antibody levels in young adults through delayed infection with, or reactivation of, each other. These suggest increased antibodies against these two viruses in MS may be interrelated.

The question remains whether a causeeffect relation exists between infectious organisms and MS, or whether viruses are just a consequence of the activation of the inflammatory-immune sequence or increased susceptibility of MS patients to infection. Studies of CD46 and other viral receptors seem warranted in MS.

B Anlar

Department of Pediatric Neurology, Hacettepe University, Ankara 06100, Turkey; banlar@hacettepe.edu.tr

\section{References}

1 Hawkes $\mathrm{CH}$. Is multiple sclerosis a sexually transmitted infection? J Neurol Neurosurg Psychiatry 2002;73:439-43.

2 Stewart G. Infection and multiple sclerosis-a new hypothesis? J Neurol Neurosurg Psychiatry 2002;73:358-9.

3 Enbom M. Human herpesvirus 6 in the pathogenesis of multiple sclerosis. APMIS 2001;109:401-11.

4 Soldan SS, Fogdell-Hahn A, Brennan MB, et al. Elevated serum and cerebrospinal fluid levels of soluble human herpesvirus type 6 cellular receptor, membrane cofactor protein, in patients with multiple sclerosis. Ann Neurol 2001;50:486-93.

5 Mason JC, Yarwood H, Sugars K, et al. Induction of decay-accelerating factor by cytokines or the membrane-attack complex protects vascular endothelial cells against complement deposition. Blood 1999;94:1673-32.

\section{Infection and multiple sclerosis}

The paper by $\mathrm{C} \mathrm{H}$ Hawkes (Is multiple sclerosis a sexually transmitted infection? $\left.{ }^{1}\right)$ has caused predictable distress to people with multiple sclerosis (MS) and their families. Living with MS is a difficult enough experience without such sudden and avoidable alarm. The UK Multiple Sclerosis Society's national helpline and local branches have been inundated with calls expressing anger and anxiety.

It is hard to understand the motive for publication when your own expert editorial commentator specifically referred to the paper's "pure speculation" and "potential to cause harm". Did the sensational nature of Dr Hawkes's hypothesis and the virtual guarantee of extensive publicity it could receive outweigh proper consideration of its scientific merit?

There is also the worrying question of what damage may have been caused to the reputation of MS research in the UK by the lay media coverage which was attracted. The MS
Society has a current forward commitment of around $£ 12$ million to nearly 70 research projects. That money is raised by voluntary donation. Anything which could discredit the quality of research here is of material concern to us.

M O'Donovan

Chief Executive, The Multiple Sclerosis Society, M S National Centre, 372 Edgware Road, London NW2 6ND, UK; modonovan@mssociety.org.uk

\section{Reference}

1 Hawkes $\mathrm{CH}$. Is multiple sclerosis a sexually

transmitted infection? J Neurol Neurosurg

Psychiatry 2002;73:439-43.

Ed: The journal regrets any distress caused to patients with MS as a result of the widespread publicity this article received in the media.

However, we wish to emphasise that the article was subject to the usual peer review process.

\section{BOOK REVIEWS}

\section{Delirium in old age}

Edited by J Lindesay, K Rockwood, A Macdonald (Pp 238, £55.00). Published by Oxford University Press, Oxford, 2002. ISBN 0-19-263275-2

Delirium is an extremely important condition for a number of reasons. It is very distressing and frightening for those who experience the symptoms, and descriptions of the effects on the brain as a result of high fever have been well described. There is a high mortality associated with the development of delirium, and it is often associated with behavioural disturbances that can be troublesome for carers and attendants. Finally, it presents a unique opportunity to look at the interface between psychiatric symptoms caused by organic disease and functional disorders.

Twelve years ago, the same publishers and two of the current editors produced the first edition on delirium. It was a relatively thin book but set the standards that the current edition continue. Delirium is certainly a niche market, and there appear to be no direct competitors, although textbooks on old age psychiatry usually contain chapters and notes on delirium. The new edition is greatly expanded and very much up to date.

Every aspect of delirium is included, from the history and conceptual basis of the disorder through epidemiology, neurophysiology, clinical assessment, management, prevention, and, refreshingly, the the role of family caregivers and nurses in managing the disease. The core tenet of the book is that delirium is a disorder that is relatively poorly recognised (particularly the hypo-alert type) by the general clinical professions, it is relatively easy to identify people at risk of developing delirium, and that there is a real possibility of a reasonable preventive strategy for the disorder. Twelve authors have contributed and, as delirium is relatively under-researched, this probably represents a significant proportion of the leading researchers in the field internationally. There are particularly interesting sections on the conceptual basis of the disorder and how it, and its component symptoms, are defined, methods of assessment of delirium are covered comprehensively, a summary of how evidence based management plans can be developed, and the prospects of prevention of delirium are given an adequate airing.

An interesting spin, which I discovered by accident, is that on the Oxford University Press website (www.oup.co.uk), one can see online updates of each individual chapter. Those present when this author last visited the website (December 2002) consisted of work that had been done from when the manuscript had been submitted to publication. It may be that reviews of the book might also appear online-this one will.

The book is a landmark in the literature on delirium, is a text of very high quality, and anyone seriously involved in the clinical management of patients with delirium or research on the subject would do very well to read this book.

A Burns

\section{Neurophysiology in neurosurgery. A modern intraoperative approach}

Edited by Vedran Deletis and Jay L Shils (Pp 469, \$125.00). Published by Academic Press, California, 2002. ISBN 0-12209036-5

This book comprises 17 chapters contributed by 24 authors. It has clearly benefited from most of the chapters being written in a more or less homogenous style and formed into seven parts based mainly on surgical procedures: motor evoked potentials/ neurophysiological base; intraoperative neurophysiology (ION) of the spinal (spinal cord monitoring); ION of peripheral nerves, nerve roots and plexuses; ION of cranial nerve and brainstem; ION of supratentorial procedures; ION during stereotactic neurosurgery for movement disorders; and ION and anaesthesia management. Most of the chapters cover the background of methodology, description of the surgical procedure, and the related neurophysiological procedure, personal experience, and case reports, which gives a balanced theoretical and practical view on the topic of each chapter. The interdisciplinary approach taken in this book will ensure it has a wide range of readers across "neurosurgery, neurology, orthopaedic surgery, neurophysiology, anesthesiology, interventional radiology, and biomedical engineering"

Chronic deep brain stimulation or neuromodulation has extended the role of clinical neurophysiology beyond its traditional diagnostic role. This new field is touched upon briefly in the part on ION during stereotactic neurosurgery. An interesting feature of this book is that it is accompanied by a CD that certainly enhances its value. Cross references are given at the end of the corresponding chapter rather than in the list of contents in the book, and at the front page of the display.

In conclusion, it is an authoritative review of intraoperative neurophysiology much weighted on the motor system for a wide range of surgical procedures. Perhaps, in its present form, those hoping for a more systematically informed discussion on intraoperative neurophysiology of the sensory system may feel slightly disappointed.

X Liu, T Z Aziz 


\section{Clinical neurophysiology of the vestibular system, 3rd edition}

Edited by R.W. Baloh and V. Honrubia (Pp 408, US\$98.50). Published by Oxford University Press, New York, 2001. ISBN 0-19513982-8.

The first edition of Clinical neurophysiology of the vestibular system, published in 1979, had a significance beyond its content: it affirmed that neurology had a stake in the vestibular system. Here was a neurologist (Baloh) writing with an otolaryngologist (Honrubia) about semicircular canals, endolymph, audiograms, and above all the vestibulo-ocular reflex-the "VOR". The VOR is no ordinary reflex; one can measure accurately both its input and its output and come up with a transfer function for gain-a new concept then for neurology. We have learnt a lot more about measurement of vestibular function and about disorders of the vestibular system since 1979. The 2nd edition, published in 1990, and now the third edition, incorporate these advances.

And what a terrific book it still is: based on concepts, packed with facts, lucidly written, and rigorously referenced. Its structure is logical and its language is clear, so that it is not only easy to search and browse but a pleasure to read from cover to cover. And it is comprehensive-no vestibular stone is left unturned.

There are four main parts, dealing in turn with: the structure and function of the vestibular system (four chapters); the clinical and laboratory evaluation of the dizzy patient (four chapters); specific diseases affecting the vestibular system (10 chapters); and the treatment of vertigo and vestibular loss (two, yes only two, chapters-but then that's neurology for you).

It's impossible to single out any one chapter they are all outstanding. For example, I particularly liked the new material in chapter one on the phylogeny of the vestibular system. Now one would have to admit that familiarity with the otocyst of the sea anemone is not a lot of use in the consulting room, but this section is so clearly written and matter so interestingly explained that one happily dispenses with such utilitarian demands.

The great strength of the book and what has made it such a classic, is that although it is based on physiology, full comprehension of physiology is not a prerequisite for retrieving useful information from the disease based chapters. Although the structure is there, one can put this aside and simply delve. The chapters on the three most common vestibular diseases, benign positional vertigo, migraine, and Meniere's diseases, are absolute gems. Each could be published as a self-contained review in its own right.

The book is an elegant conceptual and factual account of the vestibular system, its disorders and diseases, rather than a self-help or how I do it manual. Some readers might miss not having, a "frequently asked clinical questions" section, or at least a "frequently encountered clinical pitfalls" section, but then no one can have it all. Anyone who sees dizzy patients needs one dizzy book on the desk. This is the one I have on mine.

G M Halmagyi

\section{Role of proteases in the pathophysiology of neurodegenerative diseases}

Edited by Abel Lajtha and Maren L Banik (Pp 302 , £59.50). Published by Kluwer 2001. ISBN 0-306-46579-5

This volume would be an extremely useful addition to the bookshelf of anybody with an active interest in the biochemical and pathological processes that underlie some of the more common neurological diseases. In the past the role of proteolysis in these disorders has been largely neglected because it was assumed that it represented a general nonspecific metabolic process. In terms of attracting research interest the field also suffered from the confusion in the literature concerning the naming of these enzymes and the fact that the same enzyme might have many different names. However, as the editors point out in their preface, this is no longer the case and they have managed to bring together an impressive array of current research on the involvement of proteases in a wide variety of disorders. From what individually might have been regarded as rather disparate studies, one can now start to see common themes not least of which is the potential therapeutic value of targeting specific proteases and the development of specific inhibitors.

If, like me, you don't have specialist knowledge of this area I would recommend going straight to the last chapter on the mammalian proteinase genes. Here you will find a clearly laid out summary of the classification and characteristics of the four main groups of proteases (serine, cysteine, aspartic, and metalloproteinases). I also found the chapter on the ubiquitin/proteasome system and the normal physiological breakdown of proteins particularly informative. Having read these two chapters you then have a wide choice of disorders and proteases to choose from. Perhaps the most widely discussed is Alzheimer's disease, undoubtedly because of the huge advances that have been made in the understanding of the biochemical processes underlying this disease over the past 15 years. Papain-like cysteine proteases (cathepsins), caspases, calpains, and a novel metalloendopeptidase (EC 3.4.24.15) all appear to have some role in the pathology of Alzheimer's disease and may, therefore, be potential targets for drug development. There is also a group of Alzheimer's disease specific proteases that affect the processing of the amyloid precursor protein $(\alpha, \beta$, and, $\gamma$ secretase) and presenilin (presenilinase). Both of these proteins are central to the development of pathology and so these enzymes in particular are key targets for current drug company research.

Apart from the interest in Alzheimer's disease, there are other chapters covering the role of matrix metalloproteinases and calpain in the demyelination of multiple sclerosis and the key role of calpain in the pathology of traumatic brain and spinal cord injury. Further chapters describe the loss of calcium homeostasis and the subsequent pathological activation of calpain, resulting in the breakdown of key structural proteins in some neuromuscular disorders. In summary, this book has something for everyone in an area of research that holds huge promise for the future in terms of developing useful therapies for treating neurodegenerative disorders.

S Gentleman

\section{CORRECTIONS}

The following abstract was not printed with the article by E L J Hoogervorst, M J Eikelenboom, B M J Uitdehaag, and C H Polman (One year changes in disability in multiple sclerosis: neurological examination compared with patient self report) in the April issue of JNNP (JNNP 2003;74:439-42).

Objective: To characterise the relation between one year changes in neurologist rating of neurological exam abnormalities as measured by the EDSS and changes in patient perceived disability as measured by the GNDS in patients with MS

Methods: 250 patients with MS were recruited at an outpatient clinic. Disability at baseline and one year follow up was assessed using the EDSS and GNDS. Correlations between change in EDSS, GNDS-sum score, functional systems, and GNDS subcategories were studied as well as the significance of changes in EDSS associated with changes in perceived disability.

Results: The correlation between one year changes in EDSS $v$ GNDS was substantially lower $(0.19)$ than cross-sectional correlations between EDSS and GNDS, either at baseline (0.62) or at follow up (0.77). Notably, changes in functional system scores that are based on neurological examination are poorly or not at all correlated with changes in disability as perceived by the patient. Analysing the impact of a significant worsening in EDSS score we found that this was associated with significant worsening, insignificant change, and significant improvement in the patients' perceived disability in $45 \%, 39 \%$, and $15 \%$ of patients, respectively.

Conclusion: Patients' perception of change in disability differs not only quantitatively but also qualitatively from that of an examining physician. There are true differences in change as perceived by the patient and measured by the physician and changes in many dimensions of disability are relevant to the patient and have no measurable impact on the EDSS.

The authors of the short report entitled Paraneoplastic ophthalmoplegia and subacute motor axonal neuropathy associated with anti-GQlb antibodies in a patient with malignant melanoma, published in the April issue 2003 of JNNP (2003;74:507-9), were listed in the incorrect order. The author order should read as follows: L Kloos, C W Ang, W Kruit, G Stoter, and P Sillevis. 